\title{
Numerical Simulation of Magnetic Nano Drug Targeting in Patient-Specific Lower Respiratory Tract
}

\author{
Flavia Russo $^{\mathrm{a}}$, Andrea Boghi ${ }^{\mathrm{b}, *}$, Fabio Gori ${ }^{\mathrm{a}}$ \\ ${ }^{a}$ Department of Mechanical Engineering, University of Rome "Tor Vergata", Via del \\ politecnico 1, 00133 Rome, Italy \\ ${ }^{b}$ School of Water, Energy and Environment, Cranfield University, Cranfield, Bedfordshire \\ MK43 OAL, United Kingdom
}

\begin{abstract}
Magnetic nano drug targeting, with an external magnetic field, can potentially improve the drug absorption in specific locations of the body. However, the effectiveness of the procedure can be reduced due to the limitations of the magnetic field intensity. This work investigates this technique with the Computational Fluid Dynamics (CFD) approach. A single rectangular coil generates the external magnetic field. A patient-specific geometry of the Trachea, with its primary and secondary bronchi, is reconstructed from Digital Imaging and Communications in Medicine (DICOM) formatted images, throughout the Vascular Modelling Tool Kit (VMTK) software. A solver, coupling the Lagrangian dynamics of the magnetic nanoparticles with the Eulerian dynamics of the air, is used to perform the simulations. The resistive pressure, the pulsatile inlet velocity and the rectangular coil magnetic field are the boundary conditions. The dynamics of the injected particles is investigated without and with the magnetic probe. The flow field promotes particles adhesion to the tracheal wall. The particles volumetric flow rate in both cases has been calculated. The magnetic probe is shown to increase the particles flow in the target region, but at a limited extent. This behavior has been attributed to the small particle size and the probe configuration.
\end{abstract}

Keywords: Magnetic Hydro Dynamics, Patient-Specific, nanoparticles, Lagrangian model, Eulerian model, Lower respiratory tract.

\section{Introduction}

The lung cancer, a malignant tumor, is the first cause of death among common cancers, with the World Health Organization reporting a number of death of 1.5 million during 2012 [1]. The disease strikes mainly men, $16.7 \%$ of the

\footnotetext{
${ }^{2}$ Nano Drug Targeting in Lower Respiratory Tract

*A. Boghi

Email address: a.boghi@cranfield.ac.uk (Andrea Boghi)
} 
total, with the highest estimated age-standardized incidence rate in Central, Eastern Europe and Eastern Asia. The incidence is lower among women, with the highest estimated rates in Northern America and Northern Europe [2]. It appears impossible to prevent this disease, although the incidence can be reduced by avoiding the main risk factors, such as smoking and air pollution. Treatment and long term outcomes rely on the type of cancer, the stage when is treated and the patients health. Chemotherapy, surgery and radiotherapy, are the most commonly used treatments, despite their numerous hazardous side effects.

In the last decade, new technologies have been developed in order to resolve these issues. In the magnetic therapy the drugs are guided directly into the interested organs, reducing the drug absorption in the tissues which are not interested by the tumor. Lungs represent an ideal target for drug delivery, due to the direct access and the large area exposed to drugs [3]. Different types of nanoparticles to enhance drug delivery have been studied in [4]. Mouse lungs have been investigated in [5] where a large number of leukocytes have been found in the lungs parenchyma and in the bronchiole lumen, suggesting they were attracted by the magnetic nanoparticles present.

Several Computational Fluid Dynamics (CFD) studies have been carried out to investigate blood flow [6-12] and the effect of nanoparticles embedded in the air flow. Nano and micro particles deposition, after the construction of idealized airway geometries, have been studied in [13] in order to find the optimal particle diameter for drug targeting. The transport and deposition of nanoparticles for cyclic and steady flow at low Reynolds numbers, have been studied in [14] by evaluating the mass transfer due to nanoparticles dispersion. The inspiratory flow in a three-generation symmetric bifurcation, under the assumption of low Reynolds numbers has been investigated in [15], while the turbulent flow has been investigated with the $k-\omega$ model in [16]. Two breathing conditions, the resting/normal and the maximal one, have been studied in [17] by employing a patient specific geometry. The secondary flow fields and the inertial effects in patient specific lung geometries, obtained from Computed Tomography (CT) data set has been studied in [18]. Other studies focused on subject specific boundary conditions [19] and on the application of CFD to the surgery field in order to evaluate the flow rates in patients with bidirectional anastomosis [20]. The particle deposition in the lungs has been investigated in [21], by using two different geometry models in order to analyze the best regions where the deposition mechanism was higher.

Biological effects of electromagnetic fields have been investigated in [22], while the application of the magnetic technique for the transport of drugs and tracers in specific targets has been developed in [23]. Few CFD studies have been conducted to simulate the magnetic drug targeting in blood vessels both in idealized [24-27] and patient-specific $[28,29]$ geometries. A mathematical model has been proposed in [30] in order to investigate the deposition of magnetic particles aerosol in lung alveolus, by considering only one alveolus with a simplified spherical geometry. A particles diameter of $5 \mu \mathrm{m}$ and a quadrupolar Halbach permanent magnet array have been used for that study. 
The present study investigates the fluid dynamics of air inside the lower respiratory tract, where nanoparticles, used for drug targeting, are aerosolized, inhaled and dragged from the trachea to the bronchiole by an external magnetic field. A patient-specific geometry is reconstructed from a data set of CT scan images of a middle-aged healthy man. The air is treated as a continuum medium with an Eulerian formulation, while a Lagrangian approach is used for the nanoparticles. A rectangular coil is the source of the external magnetic field with a current intensity which complies with the clinical standards. The results of the simulations without and with the magnetic probe are compared.

\section{Materials and Methods}

\subsection{Domain reconstruction}

The domain's geometry is generated with the open-source software VMTK (The Vascular Modeling Toolkit) [31], which reconstructs a real chest surface, with the trachea and its primary and secondary bronchi, from a DICOM series of images. The lungs are divided into left and right, which can be further divided into upper, lower and central lobes. The geometry takes into account the principal and secondary bronchial tubes, belonging to the upper and central lobes of the right lung, whereas the interested regions of the left one are located in the lower lobe. The magnetic field is applied to the upper lobe of the right lung, where the tumor is supposed to be. Because of the chest geometry, the axis of the magnetic probe is directed towards the left side of the abdomen. Since the procedure is operator-dependent, the non-interesting structures and artifacts are removed manually. The Level Set algorithm is applied to reconstruct the surface of interest [32, 33], which is refined with the Parametric Deformable Models, initialized with the Colliding Front methodology. The output of the Level Set algorithm is an image, and the Marching Cubes algorithm is used to reconstruct the surface, due to the depth of the interested geometry.

Flow extensions of cylindrical shape, equal to 6 times its diameter, are added to the inlets and the outlets of the domain to ensure that the flow, entering and leaving the computational domain, is fully developed. This approach allows to use standard boundary conditions (BC) to solve the partial differential equations (PDE) governing the phenomenon. The computational grid is generated once the flow extensions are added. An adaptive mesh is employed with a more refined grid close to the wall and in the smaller branches. The computational grid employs tetrahedral elements, with minimum and maximum dihedral angles, set up in order to reduce the skew angle and the number of non-orthogonal cells. Four grids are generated with different number of elements, respectively 144,712 (grid1), 514,723 (grid2), 853,982 (grid3) and 1,245,423 (grid 4), to verify that the numerical solutions are grid-independent $[15,16]$.

The domain is shown in Fig.(1), where Fig.(1,a) presents the reconstruction obtained with the application of the Marching Cubes algorithm, while Fig. $(1, b)$ labels the segments whose drug uptake is monitored during the numerical simulations. 
(a)

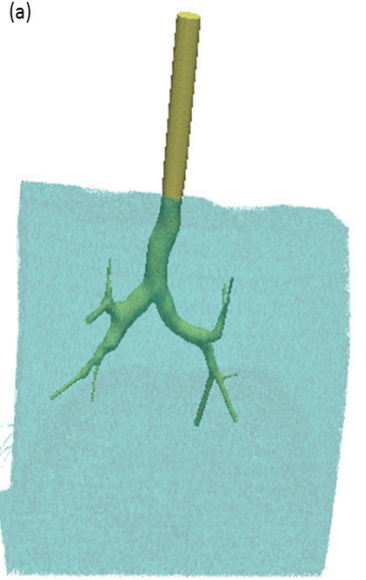

(b)

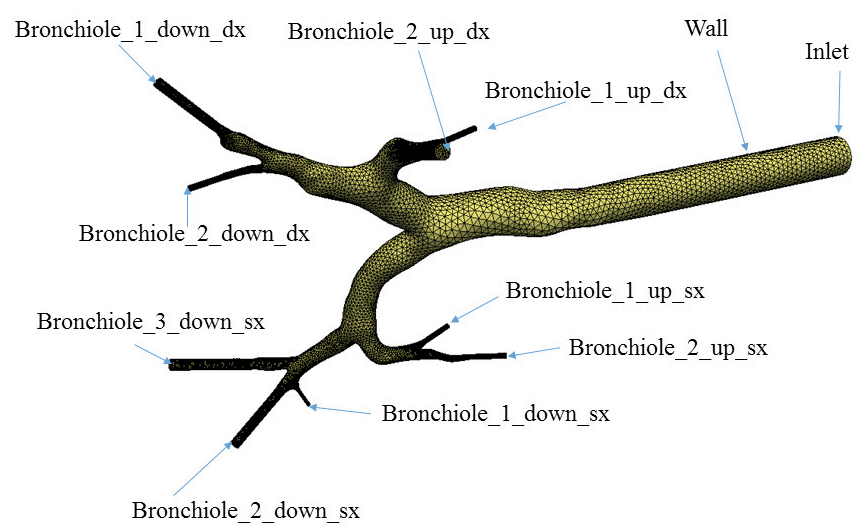

Figure 1: (a) Application of the Marching Cubes algorithm to obtain a reconstruction of the Trachea with its collateral structures; (b) Detail of the geometry used in this study.

\subsection{Eulerian Model}

The Magneto Hydro Dynamic (MHD) mathematical description is based on the coupling between the Navier-Stokes and the Maxwell equations. The MHD equations can be written for incompressible flow of air as follows:

$$
\begin{gathered}
\operatorname{div}\left(\vec{v}_{a}\right)=0 \\
\frac{\partial \vec{v}_{a}}{\partial t}+\left(\vec{v}_{a} \cdot \nabla\right) \vec{v}_{a}=-\frac{1}{\rho_{a}} \nabla p+\nu_{a} \nabla^{2} \vec{v}_{a}+\vec{g}+\frac{1}{\rho_{a} \mu_{0}} \operatorname{curl}(\vec{B}) \times \vec{B} \\
\frac{\partial \vec{B}}{\partial t}+\left(\vec{v}_{a} \cdot \nabla\right) \vec{B}=(\vec{B} \cdot \nabla) \vec{v}_{a}+\frac{1}{\sigma_{a} \mu_{0}} \nabla^{2} \vec{B}
\end{gathered}
$$

where the subscript $a$ denotes "air", $\nu_{a}$ is the kinematic viscosity, $\rho_{a}$ the density, $\mu_{0}$ the magnetic permeability in the vacuum, $\sigma_{a}$ the electric conductivity, $\vec{g}$ the gravity acceleration, $p$ the static pressure, $\vec{v}_{a}$ the velocity field, and $\vec{B}$ the magnetic induction field. As above mentioned, the air has been considered as an incompressible fluid. This is due to the fact that in physiological condition the velocity of the air is much smaller than the speed of sound and therefore the Mach number is much smaller than 1, and the fluid can be considered incompressible [34].

The air-momentum equation, Eq.(2), does not take into account the particlesair momentum transfer, because for particle volume fraction smaller than $10^{-6}$ 
the disperse phase does not influence the continuum face, in agreement with $[35,36]$. The effect of the magnetic field on the free ions and the erythrocytes is taken into account by the Lorentz force in Eq.(2).

The particle diameter used in this work is $5 \mathrm{~nm}$, which is typical of gold/ironoxide nanoparticles [37,38], and the particle geometry is spherical. Other shapes can be used, and their effect on the fluid flow is documented in the literature [39-41], but they are not considered here, because the Lagrangian model used is not suitable for non-spherical particles.

\subsection{Lagrangian Model}

Let us consider a particle of diameter $d_{p}$, velocity $\vec{v}_{p}$, and mass $m_{p}$, whose center position is $\vec{x}_{p}$. In a Lagrangian frame of reference, the position of each particle is obtained by the integration of its velocity,

$$
\frac{d \vec{x}_{p}}{d t}=\vec{v}_{p}
$$

which is evaluated from the momentum conservation equation, written as

$$
\begin{aligned}
& \frac{d \vec{v}_{p}}{d t}=-\underbrace{\frac{1}{\tau_{p}}\left(\vec{v}_{p}-\vec{v}_{a}+\frac{d_{p}^{2}}{12} \nabla^{2} \vec{v}_{a}\right)}_{(I)}+\underbrace{\left(1-\frac{\rho_{a}}{\rho_{p}}\right)}_{(I I)} \vec{g}+\underbrace{\frac{\rho_{a}}{\rho_{p}}\left(\frac{\partial \vec{v}_{a}}{\partial t}+\left(\vec{v}_{a} \cdot \nabla\right) \vec{v}_{a}\right)}_{(I I)}+ \\
& \underbrace{\frac{1}{2} \frac{\rho_{a}}{\rho_{p}}\left(\frac{\partial \vec{v}_{a}}{\partial t}+\left(\vec{v}_{a} \cdot \nabla\right) \vec{v}_{a}-\frac{d \vec{v}_{p}}{d t}\right)}_{(I V)}+\underbrace{\left(\frac{q_{p}}{m_{p}} \vec{v}_{p}-\frac{1}{\rho_{p} \mu_{0}} \operatorname{curl}(\vec{B})\right) \times \vec{B}}_{(V)}
\end{aligned}
$$

where $\rho_{p}=6 m_{p} / \pi d_{p}^{3},(I)$ is the drag, $(I I)$ the buoyancy, $(I I I)$ the carrier phase inertia, $(I V)$ the added mass and $(V)$ the Lorentz force. The particleparticle interactions is neglected in Eq.(5) because of the small volume of the particles, which reduces the probability of collision. Furthermore, $q_{p}$ is the electric charge of the particle and $\tau_{p}$ the relaxation time, defined as

$$
\tau_{p}=\frac{4}{3} \frac{\rho_{p} d_{p}}{\rho_{b} C_{d}\left|\vec{v}_{b}-\vec{v}_{p}\right|}
$$

The standard definition of the drag coefficient, according to [42], is the following

$$
C_{d}= \begin{cases}\frac{24}{\operatorname{Re}_{\mathrm{p}}} & \operatorname{Re}_{\mathrm{p}}<0.1 \\ \frac{24}{\operatorname{Re}_{\mathrm{p}}}\left(1+\frac{1}{6} \operatorname{Re}_{\mathrm{p}}^{2 / 3}\right) & 0.1<\operatorname{Re}_{\mathrm{p}}<1000 \\ 0.44 & \operatorname{Re}_{\mathrm{p}}>1000\end{cases}
$$

where the particle Reynolds number is defined as

$$
\operatorname{Re}_{p}=\frac{d_{p}\left|\vec{v}_{b}-\vec{v}_{p}\right|}{\nu_{b}}
$$




\subsection{Boundary Conditions (BC)}

The solution of the Eulerian system requires appropriate boundary conditions (BC). A non-slip BC is imposed for the velocity on the wall. As far as the outlets are concerned, a mixed BC is employed: when the air leaves the domain the velocity normal derivative is set to zero, whereas the tangential velocity is set to zero when air enters through the boundary. The velocity profile is imposed on the inlet with a parabolic profile in steady state, while a Womersley-Evans profile is employed in unsteady state, in analogy with [10, 29],

$$
v_{a}(t, \xi)=8 \frac{Q}{\pi D^{2}}\left(1-\xi^{2}\right)+2 \Re\left(\sum_{n=1}^{N} V_{n} \Phi\left(\tau_{n}, \xi\right) e^{j \omega_{n} t}\right)
$$

where

$$
\Phi\left(\tau_{n}, \xi\right)=\frac{J_{0}\left(\tau_{n}\right)-J_{0}\left(\tau_{n} \xi\right)}{J_{0}\left(\tau_{n}\right)-2 J_{1}\left(\tau_{n}\right) / \tau_{n}}
$$

and

$$
\tau_{n}=j^{\frac{3}{2}} \frac{D}{2} \sqrt{\frac{\rho}{\mu_{\infty}} \omega_{n}}=j^{\frac{3}{2}} \alpha_{n}
$$

Being $r$ the radial coordinate, $D$ the tracheal diameter, $Q$ the volumetric flow rate, $\xi=2 r / D, J_{0}$ and $J_{1}$ the zeroth and first-order Bessel functions of the first kind, $\alpha_{n}$ the Womersley numbers of order $n, \Re()$ the real part of a complex number, $j=\sqrt{-1}, V_{n}$ the Fourier coefficients of the pulsatile mean velocity profile and the number of harmonics used to reproduce the flow rate. By using the Fast Fourier Transform (FFT) algorithm, the first ten Fourier coefficients of the flow rate in the trachea, derived from experimental data [17-19], are employed to reconstruct the velocity profile.

The physiological waveform is reported in Fig.(2). The flow rate is pulsatile and the Reynolds number varies from 0 to 2536, meaning that the flow is laminar mostly and becomes transitional only at the peaks of the inhalation and exhalation phases. For this reason the flow is treated as laminar, in agreement with $[14,15]$.

As far as the pressure in the unsteady state is concerned, the resistive BC, derived in $[9,20]$, is imposed on all the outlets

$$
p=p_{a}+R Q
$$

being $p_{a}$ the reference pressure in the alveoli and $R$ the hydraulic resistance. The value of the resistance and the reference pressure are extrapolated from the steady state simulations by imposing the volumetric flow rates and a zero normal derivative condition for the pressure on the outlets. 


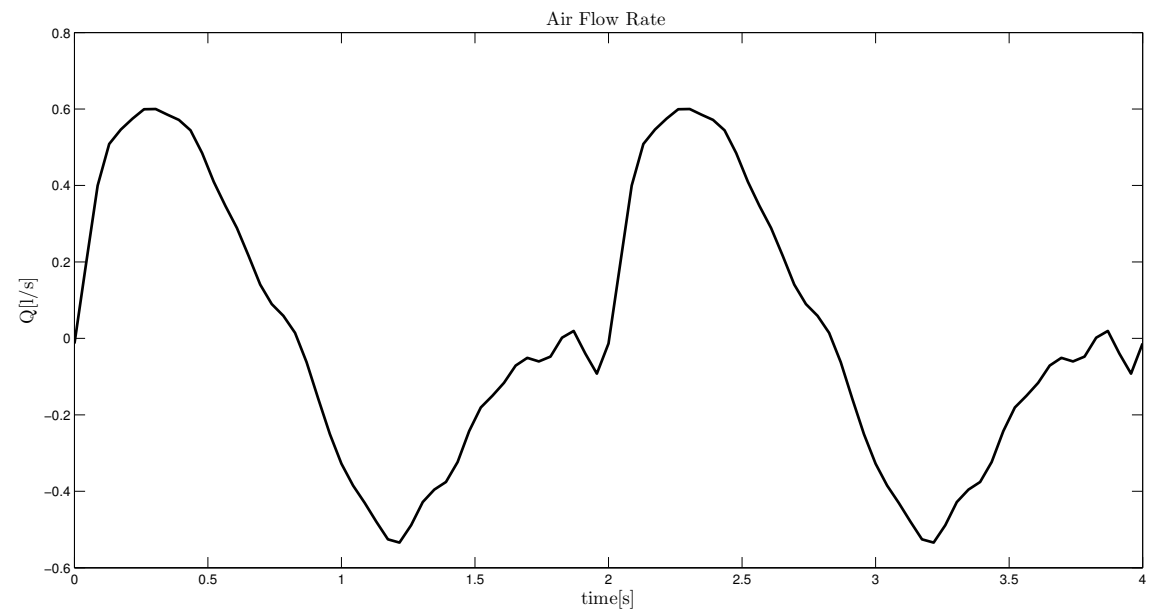

Figure 2: Pulmonary Flow Rate vs time.

\subsection{Magnetic Induction $B C$}

The Boundary Conditions for the magnetic induction field are zero normal derivatives everywhere, except on the wall, where the magnetic field of the probe is imposed. The external magnetic field is generated by a single rectangular coil, with a negligible cross section of the wire, where an electric current is flowing. This single rectangular coil is quite common in the clinical practice [43-46].

An analytical expression for the magnetic induction field is derived in [47]. A point in the coil reference frame, whose origin is at its centre, is identified by the coordinates $\left(x^{\prime}, y^{\prime}, z^{\prime}\right)$. The coil dimensions are $2 a_{1}$ along the $x^{\prime}$ axis and $2 b_{1}$ in the $y^{\prime}$ direction, with a section of $2.5 \mathrm{~cm}^{2}$. The axis is normal of the coil surface. The axis $z^{\prime}$ is normal of the coil surface. The components of the magnetic induction field are

$$
\begin{gathered}
B_{x^{\prime}}=\frac{\mu_{0} I_{1}}{4 \pi} \sum_{a=1}^{4}\left[\frac{z^{\prime}(-1)^{a+1}}{r_{a}\left[r_{a}+d_{a}\right]}\right] \\
B_{y^{\prime}}=\frac{\mu_{0} I_{1}}{4 \pi} \sum_{a=1}^{4}\left[\frac{z^{\prime}(-1)^{a+1}}{r_{a}\left[r_{a}+C_{a}(-1)^{a+1}\right]}\right] \\
B_{z^{\prime}}=\frac{\mu_{0} I_{1}}{4 \pi} \sum_{a=1}^{4}\left[\frac{d_{a}(-1)^{a}}{r_{a}\left[r_{a}+C_{a}(-1)^{a+1}\right]}-\frac{C_{a}}{r_{a}\left[r_{a}+d_{a}\right]}\right]
\end{gathered}
$$

with 


\begin{tabular}{cccc}
\hline Normal & CoilCoords $(\mathrm{m})$ & TargetCoords $(\mathrm{m})$ & $\left.\vec{B}\right|_{1 \mathrm{~cm}}(\mathrm{mT})$ \\
\hline$n_{x}=-0.082166$ & $x_{c}=-0.054377$ & $x_{t}=0.0314$ & 0 \\
$n_{y}=0.93057$ & $y_{c}=-0.0891$ & $y_{t}=-0.3554$ & 0 \\
$n_{z}=0.35677$ & $z_{c}=-0.21683$ & $z_{t}=0.9342$ & 46.21 \\
\hline
\end{tabular}

Table 1: Source and target coordinates; values for the normal to the chest surface of the patient; intensity of the Magnetic field along the $z^{\prime}$ - axis.

\begin{tabular}{cccccc}
\hline$H_{D}(\mathrm{~cm})$ & $W_{D}(\mathrm{~cm})$ & $D_{D}(\mathrm{~cm})$ & $D_{M-T}(\mathrm{~cm})$ & $T_{O}(\mathrm{~s})$ & $\mathrm{Re}_{\max }$ \\
\hline 31.6 & 10.0 & 2.0 & 12.32 & 2.0 & 2536
\end{tabular}

Table 2: Simulation Parameters: $H_{D}$ (domain height), $W_{D}$ (domain width), $D_{D}$ (domain depth), $D_{S-T}$ (magnet-tumor distance), $T_{O}$ (observation time), Re $\mathrm{max}_{\text {max }}$ (max tracheal Reynolds number).

$$
\left\{\begin{array}{c}
C_{1}=-C_{4}=a_{1}+x^{\prime} \\
C_{2}=-C_{3}=a_{1}-x^{\prime} \\
d_{1}=d_{2}=y^{\prime}+b_{1} \\
d_{3}=d_{4}=y^{\prime}-b_{1} \\
r_{a}=\sqrt{C_{a}^{2}+d_{a}^{2}+z^{\prime 2}}
\end{array}\right.
$$

The magnetic probe is located $1 \mathrm{~cm}$ above the patient skin, in order that its modulus is smaller than $1.5 \mathrm{~T}$. This is the limit allowed in clinical treatments $[3,4]$, since higher values can cause damage to the patient. The centre of the probe and the target are aligned with the $z^{\prime}$ axis. The target of the magnetotherapy is located at the upper lobe of the right lung because the maximum magnetic field must be concentrated on it.

The external chest surface of the patient is reconstructed using VMTK, with the procedure previously illustrated for the trachea. Two geometries are located in the same reference frame, in order to calculate the coordinates of the probe and the target. The dimensions of the rectangular coil, the current intensity and the positions of coil and target are reported in Tab.(1), while the main simulation parameters are listed in Tab.(2).

\subsection{Numerical Details}

The numerical simulations are performed with the software OpenFOAM, which solves the governing equations through the Finite Volume Method (FVM). The present problem has been solved with the mhdB4Foam solver, developed in [29], which couples the Lagrangian particle dynamics with the Eulerian MHD. Some Boundary Conditions, such as the resistive BC, the pulsatile profile on the inlet for unsteady state and the parabolic profile for steady state, are implemented through the utility groovyBC, in analogy with [29]. Furthermore, the 
imposed magnetic field on the domain has been set using the rectMagProbe external OpenFOAM module, developed in [29]. The simulations are carried on for 2 respiratory cycles, considering a period of 1 s and a variable time step, in order to guarantee a Courant number smaller than 0.5 during the respiratory cycle.

\section{Results and Discussion}

\subsection{Steady State}

The steady state simulations are carried out until convergence is reached with the simpleFoam solver of OpenFOAM, which solves the Navier-Stokes equations in steady state. The numerical results, obtained with the four meshes, grid1, grid2, grid3 and grid4, are compared by using the mapFields utility, which maps the fields from one grid to another. The wall shear stress (WSS), defined as

$\tau_{\text {wall }}=\hat{i}_{\text {axis }} \cdot \rho_{a} \nu_{a}\left([I]-\hat{n}_{\text {wall }} \otimes \hat{n}_{\text {wall }}\right)\left(\left[\nabla \vec{v}_{a}\right]+\left[\nabla \vec{v}_{a}\right]^{T}-\frac{2}{3} \operatorname{div}\left(\vec{v}_{a}\right)[I]\right)_{\text {wall }} \hat{n}_{\text {wall }}$

is used to evaluate the grid independence. The contours of WSS are shown in Fig.(3). The WSS does not change significantly from grid1 to grid4. Therefore, grid2 is employed to perform the unsteady simulations, being a compromise between speed of execution and accuracy.

Figure 3 shows smaller WSS in the Trachea, due to the small velocity gradient, growing in the primary bronchi and reaching the maximum in the secondary bronchi. At the entrance of the secondary bronchi, the WSS increases, due to the section reduction. In a circular pipe the WSS is inversely proportional to the cubed radius. The increased number of branches reduces the mean velocity, which tends to reduce the WSS as well. However, the section reduction between primary and secondary bronchi is such that the WSS increases, despite the smaller mean velocity in the branches.

\subsection{Unsteady State without external magnetic field}

The unsteady state simulations, without external magnetic field, are carried out by imposing the value of the current intensity to zero. As far as the inlet velocity is concerned, a pulsatile velocity profile is imposed. Figure 4 shows the time variations of the pressure field, on the domain wall, at four different time steps of the respiratory cycle.

The pressure variations during the respiratory cycle are very small, about $1 \mathrm{~cm}$ of $\mathrm{H}_{2} \mathrm{O}$. During the inhalation there is a net flux of air to the lungs, as consequence of the reverse pressure gradient directed from the environment to the lungs, which decreases the pressure from the trachea to the secondary bronchi, as shown in Fig. $(4, a)$. At the end of the inhalation, there is no net mass flow of air between lungs and environment, and the pressure is uniform, as shown in Fig.(4,b). At the end of the inspiratory phase, Fig. $(4, c)$, the pressure 

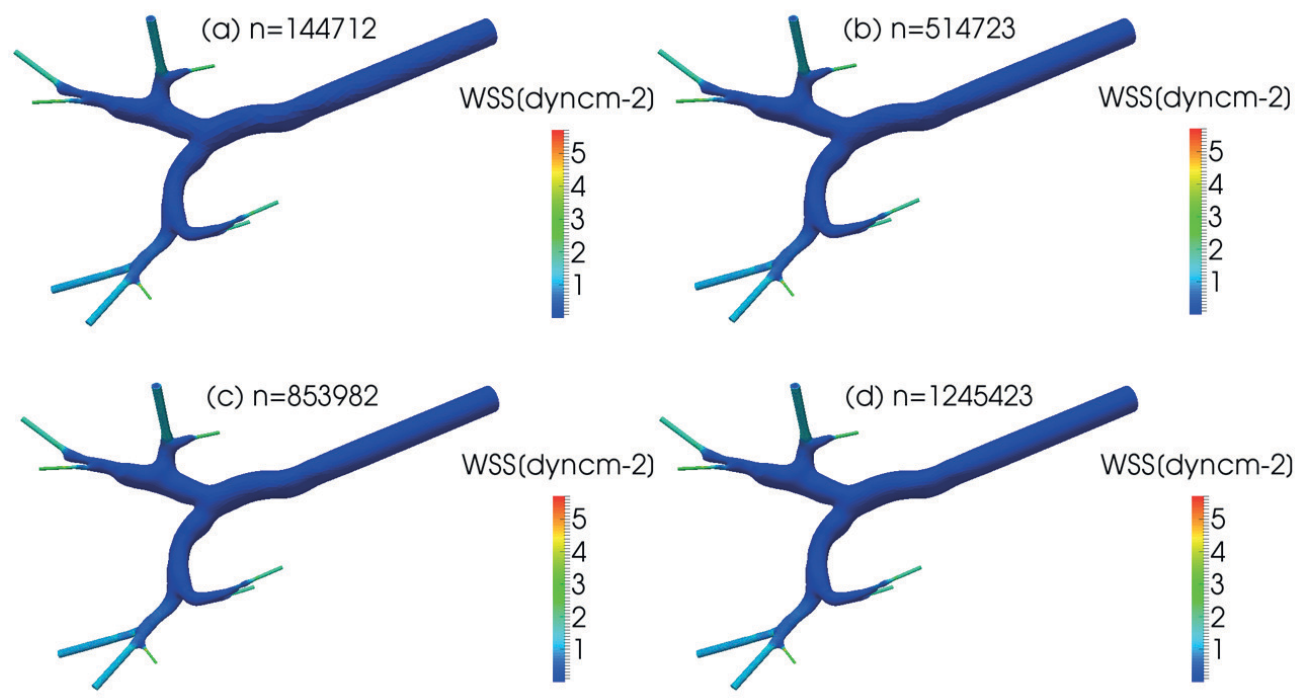

Figure 3: WSS field for the grid independence study in steady state conditions.

increases slightly. At the beginning of the exhalation, a reverse pressure gradient is established between the trachea and the secondary bronchi and air is exhaled, as shown in Fig. $(4, d)$.

Figure 5 presents the nanoparticles coloured by their speed modulus. In Fig. $(5, a-b)$ shows a parabolic-like profile at the beginning of the inhalation, confirming the laminar regime of motion, is shown. The particles with high speed leave the trachea reaching the bronchi, while the particles with low velocity tend to move towards the wall, where they are adsorbed. The adsorption of magnetic nanoparticles in bronchiole lumen has been observed in animal studies [5]. The particles adhesion to the wall is favoured by the low flow rate during the exhalation phase, as confirmed by Fig.(5,d-f).

\subsection{Unsteady State with external magnetic field}

The results of the particle motion in the respiratory system with the magnetic probe turned on are presented in this section. The position of the coil and the target, the probe direction and the intensity of the magnetic induction field, evaluated at $1 \mathrm{~cm}$ from the center of the coil, are reported in Table 1.

The maps of the magnetic field on the wall of the lower respiratory tract are shown in Figure ?? at different time steps from the beginning, up to the end 


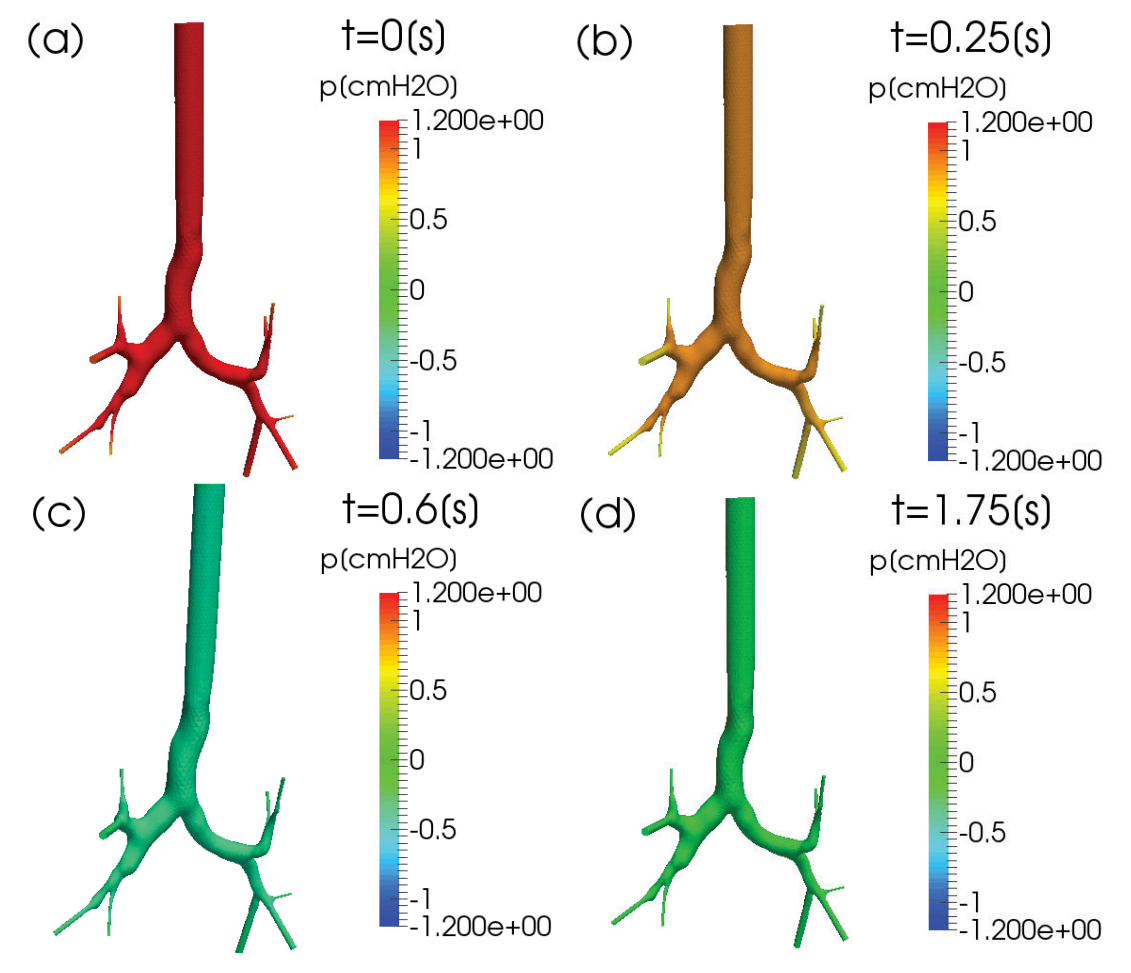

Figure 4: Unsteady Pressure field at different time-steps in absence of external magnetic field.

of the exhalation process, when the particles have filled the entire domain. The maximum value of the magnetic induction field is located on the closer branch to the right upper lobe, where the probe is located, and where the particles tend to be dragged.

The particles path-lines at six instants of time are reported in Figure 7, showing that the flow is transitional. At the beginning of the inhalation, the path-lines are mostly straight, with the presence of few vortexes, especially near the bifurcations, where the velocity gradient becomes higher because of the cross-section reduction. The highest velocity is reached in the trachea, where the environmental air enters into the lungs. Intermittent vortexes form near the bifurcations, due to the curvature changes. At the beginning of the exhalation phase, Fig.(7,b-f), the flow rate is lower and directed towards the trachea, promoting an helical flow with highly tangled path-lines. With this flow-field the time that the particles spend close to the lower respiratory tract wall increases, promoting adsorption. Since the tumor is located in the upper right lobe of the lungs the increased wall adsorption ultimately reduces the efficiency of the technique. 


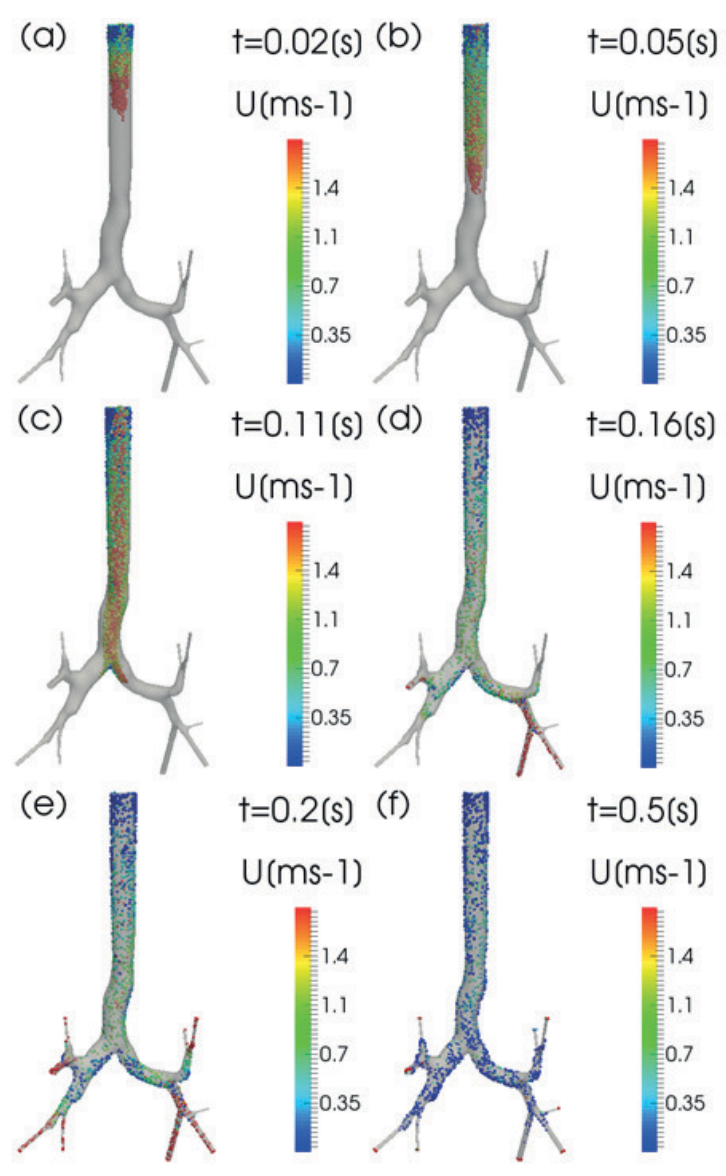

Figure 5: Particles speed at different time-steps in absence of external magnetic field.

Figures 8 and 9 show the influence of the magnetic field on the particles absorption. In order to underline the effect of the magnetic field, the results with the magnetic probe turned off are reported as well.

Figure 8 reports the particle flow rate per unit volume on the different boundaries during the respiratory cycle. In the Trachea and on the domains wall the particle flow rates are of the same order of magnitude. The peak of the exhalation process occurs between $1 \mathrm{~s}$ and $1.4 \mathrm{~s}$, when the highest flow rate from the lungs to the environment occurs. Figures $(9, \mathrm{~b}, \mathrm{e}, \mathrm{f}, \mathrm{h}, \mathrm{j})$, show that this phase corresponds to an increase in particle uptake in bronchioles, probably due to the formation of several low speed recirculation regions during the exhalation. The geometry of the bronchi is such that their diameter decreases as they proceed from the trachea to the alveoli. Therefore, since during exhalation the air moves 
from the alveoli to the trachea, a flow detachment occurs, causing recirculation regions and promoting the uptake of nanoparticles.

Since the particle flow rate may not show the effectiveness of the use of a rectangular coil in the magnetic targeting, its time integration, corresponding to the total number of particles that crosses a given section from the beginning of the process, is performed.

The probe is pointed towards the upper right lobe of the patients lungs. The uptake of nanoparticles increases in the branches of the lower respiratory tract bended in the frontal direction, Fig.( $(9, b, e, f, h, j)$, while it decreases in the branches bended towards the spine, Fig. (9,c,d,g,i). A slight reduction in the particles adhesion to the domain wall is also evidenced.

Despite the increase of particles concentration in the targeted regions, shown in Fig. $(9, b)$, the effect of the magnetic field is not significant. More than $50 \%$ of the injected particles adhere to the domain wall when the magnetic probe is turned off, while it is only $47 \%$, when the magnetic probe is turned on. Similarly, the increase of the nanoparticles in the different regions is less than $1 \%$.

This behavior can be due to the high surface-volume ratio of the lower respiratory tract, which inherently increases the probability of adhesion, regardless of the intensity of the magnetic field, and to the geometry of the probe. The magnetic field on the axis of a single square loop decreases quickly from its origin, about 100 times at a distance equal to 2.5 times its width. Considering that the distance between the source and the target is about $12.32 \mathrm{~cm}$, it is clear that this makes the modulus of the magnetic induction considerably low.

\section{Conclusions}

The present work investigates the dynamics of nanoparticles in air flow during magnetic therapy of a lung tumor, which is an emerging alternative to chemotherapy, because of the reduced side effects. At the best of the authors knowledge, this work represents the first numerical investigation of this technique in a patient-specific lower respiratory tract. The lack of numerical studies is probably due to the complexity of the problem, which couples the air flow with the nanoparticles dynamics under the influence of an external magnetic field. The purpose of this work is to verify numerically the effectiveness of this technique in the treatment of lung cancer. As every numerical study, this work is subject to the limitations due to the input parameters and the model chosen.

The numerical simulations, for steady and unsteady flow, are carried out with the OpenFOAM code in laminar flow, in agreement with $[14,15]$, with Newtonian viscosity for the air and absence of inter and intraparticle forces, due to the small size of the drug carriers. The simulations are performed in a patient specific geometry, reconstructed from CT slices with VMTK. Three routines are employed in OpenFOAM to implement the BCs for the resistive pressure, the periodic pulsatile velocity profile and the magnetic field from the rectangular coil. Furthermore, a solver coupling the Eulerian MHD of the air with the Lagrangian motion of particles is developed. 
One simulation with the magnetic probe turned off and one with the probe turn on are carried out. The results are compared to assess the increase of drug uptake in the lung due to the magneto-therapy and consequently the reduced dispersion in other locations. The rectangular coil, pointing perpendicularly to the tumor is positioned in a specific point, $1 \mathrm{~cm}$ above the upper right lobe of the lungs.

The results of the numerical simulations show that, despite the induced magnetic field increases the particle uptake, a small fraction of the total number of particles injected reaches the target. This can be due to the high surface volume ratio of the lower respiratory tract and the design of the magnetic probe.

This conclusion is in contrast with the mathematical model developed for lung alveolus in [30]. This is probably due to the different probe employed, the larger particle diameter $(5 \mu \mathrm{m})$ and the higher magnetic field $(0.2-2.2 \mathrm{~T})$. Magnetic drug delivery is extremely sensitive to these parameters. Moreover, the drug distribution in different branches is not investigated in [30]. The low performance of the magnetic drug delivery in the present study can be due to three factors: (i) the nanoparticle size; (ii) the magnet-tumor distance; (iii) the probe design. As far as the nanoparticle size is concerned, as shown by Eqs. $(18,19)$ in [29] a low particle diameter increases the acceleration due to Lorentz force, but it also increases the friction. However, because of the low magnetic field applied $[48,49]$ the effect on the friction is predominant. Therefore, larger particles should increase the effectiveness of the technique, as in [30]. The probe-tumor distance is another factor, which limits the effectiveness of the technique. Unfortunately, this parameter cannot be tuned at will, and in a superficial tumor, the magnetic drug targeting is likely be more effective.

The design of the magnetic probe is another factor that influences the outcome of the procedure. The modulus of the magnetic induction field of a single rectangular coil decreases considerably with the distance from the target, as in the right lung. Being the right lung about $12.32 \mathrm{~cm}$ far from the source, the modulus of the magnetic induction field is considerably small. A solution to this problem could be to increase the value of the current intensity flowing in the probe, but this is not possible because higher values of the magnetic field could affect the health of the patient, and is forbidden by law [48, 49]. A solution could be to modify the design of the probe in order to reduce the rate of decay of the magnetic field. A possible configuration could include multiple probes, positioned in appropriate locations.

CFD allows detailed visualization of biological fluid flows, which increases our understanding of natural phenomena, but it has some limitations due to computational resources required to simulate a process in a complex domain. The question then rises as to whether or not the observation time (2 s) is sufficient to judge the effectiveness of the technique. It would be certainly better to extend the simulations to few minutes, but the particle volumetric flow rate in Fig. 9 show negligible differences between the cases. It seems unlikely that the percentage of injected particles, which reach the right lung will significantly increase over time.

Further numerical simulations, in different patient-specific geometries and 
with different probes, are planned to assess the effectiveness of the therapy in different conditions.

\section{Acknowledgments}

This research did not receive any specific grant from funding agencies in the public, commercial, or not-for-profit sectors. The authors thank the staff of the Policlinico di Tor Vergata for the support with the biomedical images.

\section{References}

[1] D. M. Parkin, F. Bray, J. Ferlay, P. Pisani, Global cancer statistics, 2002, CA: a cancer journal for clinicians 55 (2005) 74-108.

[2] I. Globocan, Estimated cancer incidence, mortality and prevalence worldwide in 2012, 2012, (accessed 18 November 2017). http://globocan.iarc . fr/Pages/fact_sheets_cancer.aspx.

[3] N. Labiris, M. Dolovich, Pulmonary drug delivery. part i: physiological factors affecting therapeutic effectiveness of aerosolized medications, British journal of clinical pharmacology 56 (2003) 588-599.

[4] V. Chandolu, C. R Dass, Treatment of lung cancer using nanoparticle drug delivery systems, Current drug discovery technologies 10 (2013) 170-176.

[5] M. P. Garcia, R. M. Parca, S. B. Chaves, L. P. Silva, A. D. Santos, Z. G. M. Lacava, P. C. Morais, R. B. Azevedo, Morphological analysis of mouse lungs after treatment with magnetite-based magnetic fluid stabilized with dmsa, Journal of magnetism and magnetic materials 293 (2005) 277-282.

[6] F. Gori, A. Boghi, M. Amitrano, Three-dimensional numerical simulation of the fluid dynamics in a coronary stent, in: ASME International Mechanical Engineering Congress and Exposition, Proceedings, volume 2, 2009, pp. 407-411.

[7] F. Gori, A. Boghi, Image-based computational fluid dynamics in a carotid artery, in: ASME International Mechanical Engineering Congress and Exposition, Proceedings, volume 2, 2009, pp. 123-128.

[8] F. Gori, A. Boghi, Three-dimensional numerical simulation of nonnewtonian blood in two coronary stents, in: 2010 14th International Heat Transfer Conference, American Society of Mechanical Engineers, 2010, pp. 109-114.

[9] F. Gori, A. Boghi, Three-dimensional numerical simulation of blood flow in two coronary stents, Numerical Heat Transfer, Part A: Applications 59 (2011) 231-246. 
[10] A. Boghi, F. Gori, Numerical simulation of blood flow through different stents in stenosed and non-stenosed vessels, Numerical Heat Transfer, Part A: Applications 68 (2015) 225-242.

[11] I. Di Venuta, A. Boghi, F. Gori, Three-dimensional numerical simulation of a failed coronary stent implant at different degrees of residual stenosis. part i: Fluid dynamics and shear stress on the vascular wall, Numerical Heat Transfer, Part A: Applications 71 (2017) 638-652.

[12] A. Boghi, I. Di Venuta, F. Gori, Three-dimensional numerical simulation of a failed coronary stent implant at different degrees of residual stenosis. part ii: Apparent viscosity and wall permeability, Numerical Heat Transfer, Part A: Applications 71 (2017) 653-665.

[13] C. Kleinstreuer, Z. Zhang, Z. Li, Modeling airflow and particle transport/deposition in pulmonary airways, Respiratory physiology \& neurobiology 163 (2008) 128-138.

[14] Z. Zhang, C. Kleinstreuer, Airflow structures and nano-particle deposition in a human upper airway model, Journal of computational physics 198 (2004) 178-210.

[15] Y. Liu, R. So, C. Zhang, Modeling the bifurcating flow in an asymmetric human lung airway, Journal of biomechanics 36 (2003) 951-959.

[16] H. Luo, Y. Liu, Modeling the bifurcating flow in a ct-scanned human lung airway, Journal of Biomechanics 41 (2008) 2681-2688.

[17] R. Calay, J. Kurujareon, A. E. Holdø, Numerical simulation of respiratory flow patterns within human lung, Respiratory physiology \& neurobiology 130 (2002) 201-221.

[18] J. De Backer, W. Vos, C. Gorle, P. Germonpré, B. Partoens, F. Wuyts, P. M. Parizel, W. De Backer, Flow analyses in the lower airways: patientspecific model and boundary conditions, Medical engineering \& physics 30 (2008) 872-879.

[19] Y. Yin, J. Choi, E. A. Hoffman, M. H. Tawhai, C.-L. Lin, Simulation of pulmonary air flow with a subject-specific boundary condition, Journal of biomechanics 43 (2010) 2159-2163.

[20] G. Pennati, C. Corsini, D. Cosentino, T.-Y. Hsia, V. S. Luisi, G. Dubini, F. Migliavacca, Boundary conditions of patient-specific fluid dynamics modelling of cavopulmonary connections: possible adaptation of pulmonary resistances results in a critical issue for a virtual surgical planning, Interface Focus 1 (2011) 297-307.

[21] N. Nowak, P. P. Kakade, A. V. Annapragada, Computational fluid dynamics simulation of airflow and aerosol deposition in human lungs, Annals of biomedical engineering 31 (2003) 374-390. 
[22] W. R. Adey, Biological effects of electromagnetic fields, Journal of cellular biochemistry 51 (1993) 410-416.

[23] Z. Saiyed, S. Telang, C. Ramchand, Application of magnetic techniques in the field of drug discovery and biomedicine, BioMagnetic Research and Technology 1 (2003) 1-8.

[24] M. Larimi, A. Ramiar, A. Ranjbar, Numerical simulation of magnetic nanoparticles targeting in a bifurcation vessel, Journal of Magnetism and Magnetic Materials 362 (2014) 58-71.

[25] M. D. Tehrani, J.-H. Yoon, M. O. Kim, J. Yoon, A novel scheme for nanoparticle steering in blood vessels using a functionalized magnetic field, IEEE Transactions on Biomedical Engineering 62 (2015) 303-313.

[26] M. Larimi, A. Ramiar, A. Ranjbar, Numerical simulation of magnetic drug targeting with eulerian-lagrangian model and effect of viscosity modification due to diabetics, Applied Mathematics and Mechanics 37 (2016) $1631-1646$.

[27] M. Momeni Larimi, A. Ramiar, A. A. Ranjbar, Magnetic nanoparticles and blood flow behavior in non-newtonian pulsating flow within the carotid artery in drug delivery application, Proceedings of the Institution of Mechanical Engineers, Part H: Journal of Engineering in Medicine 230 (2016) 876-891.

[28] S. Kenjereš, B. Righolt, Simulations of magnetic capturing of drug carriers in the brain vascular system, International Journal of Heat and Fluid Flow 35 (2012) 68-75.

[29] A. Boghi, F. Russo, F. Gori, Numerical simulation of magnetic nano drug targeting in a patient-specific coeliac trunk, Journal of Magnetism and Magnetic Materials 437 (2017) 86-97.

[30] A. Krafcik, P. Babinec, I. Frollo, Computational analysis of magnetic field induced deposition of magnetic particles in lung alveolus in comparison to deposition produced with viscous drag and gravitational force, Journal of Magnetism and Magnetic Materials 380 (2015) 46-53.

[31] D. A. Steinman, Image-based computational fluid dynamics modeling in realistic arterial geometries, Annals of biomedical engineering 30 (2002) 483-497.

[32] L. Antiga, B. Ene-Iordache, L. Caverni, G. P. Cornalba, A. Remuzzi, Geometric reconstruction for computational mesh generation of arterial bifurcations from ct angiography, Computerized Medical Imaging and Graphics 26 (2002) 227-235. 
[33] L. Antiga, M. Piccinelli, L. Botti, B. Ene-Iordache, A. Remuzzi, D. A. Steinman, An image-based modeling framework for patient-specific computational hemodynamics, Medical \& biological engineering \& computing 46 (2008) 1097-1112.

[34] D. F. Young, B. R. Munson, T. H. Okiishi, W. W. Huebsch, A brief introduction to fluid mechanics, John Wiley \& Sons, 2010.

[35] S. Elghobashi, On predicting particle-laden turbulent flows, Applied scientific research 52 (1994) 309-329.

[36] E. Tzirtzilakis, V. Sakalis, N. Kafoussias, P. Hatzikonstantinou, Biomagnetic fluid flow in a $3 \mathrm{~d}$ rectangular duct, International Journal for Numerical Methods in Fluids 44 (2004) 1279-1298.

[37] M. Arruebo, R. Fernández-Pacheco, M. R. Ibarra, J. Santamaría, Magnetic nanoparticles for drug delivery, Nano today 2 (2007) 22-32.

[38] S. Seino, Y. Matsuoka, T. Kinoshita, T. Nakagawa, T. A. Yamamoto, Dispersibility improvement of gold/iron-oxide composite nanoparticles by polyethylenimine modification, Journal of Magnetism and Magnetic Materials 321 (2009) 1404-1407.

[39] M. Hassan, A. Zeeshan, A. Majeed, R. Ellahi, Particle shape effects on ferrofuids flow and heat transfer under influence of low oscillating magnetic field, Journal of Magnetism and Magnetic Materials 443 (2017) 36-44.

[40] A. Zeeshan, M. Hassan, R. Ellahi, M. Nawaz, Shape effect of nanosize particles in unsteady mixed convection flow of nanofluid over disk with entropy generation, volume 231, SAGE Publications Sage UK: London, England, 2017, pp. 871-879.

[41] A. Majeed, A. Zeeshan, T. Hayat, Analysis of magnetic properties of nanoparticles due to applied magnetic dipole in aqueous medium with momentum slip condition, Neural Computing and Applications (2017) 1-9.

[42] L. Schiller, Z. Naumann, A drag coefficient correlation, Vdi Zeitung 77 (1935) 51.

[43] G. J. Metzger, P.-F. van de Moortele, C. Akgun, C. J. Snyder, S. Moeller, J. Strupp, P. Andersen, D. Shrivastava, T. Vaughan, K. Ugurbil, et al., Performance of external and internal coil configurations for prostate investigations at $7 \mathrm{t}$, Magnetic resonance in medicine 64 (2010) 1625-1639.

[44] F. Dughiero, E. Baake, M. Forzan, V. Nemkov, R. Ruffini, R. Goldstein, J. Jackowski, T. DeWeese, R. Ivkov, Magnetic field generating inductor for cancer hyperthermia research, COMPEL - The international journal for computation and mathematics in electrical and electronic engineering 30 (2011) 1626-1636. 
[45] A. S. Lübbe, C. Alexiou, C. Bergemann, Clinical applications of magnetic drug targeting, Journal of Surgical Research 95 (2001) 200-206.

[46] A. S. Lübbe, C. Bergemann, H. Riess, F. Schriever, P. Reichardt, K. Possinger, M. Matthias, B. Dörken, F. Herrmann, R. Gürtler, et al., Clinical experiences with magnetic drug targeting: a phase i study with 4epidoxorubicin in 14 patients with advanced solid tumors, Cancer research 56 (1996) 4686-4693.

[47] M. Misakian, Equations for the magnetic field produced by one or more rectangular loops of wire in the same plane, Journal of research of the National Institute of Standards and Technology 105 (2000) 557-564.

[48] E. Council, Recommendation on the limitation of exposure of the general public to electromagnetic field (0 hz to $300 \mathrm{ghz})$, Official Journal of the European Communities 199 (1999) 59-70.

[49] F. C. Commission, Evaluating compliance with FCC guidelines for human exposure to radiofrequency electromagnetic fields, volume 65, OET Bullettin, 1997. 


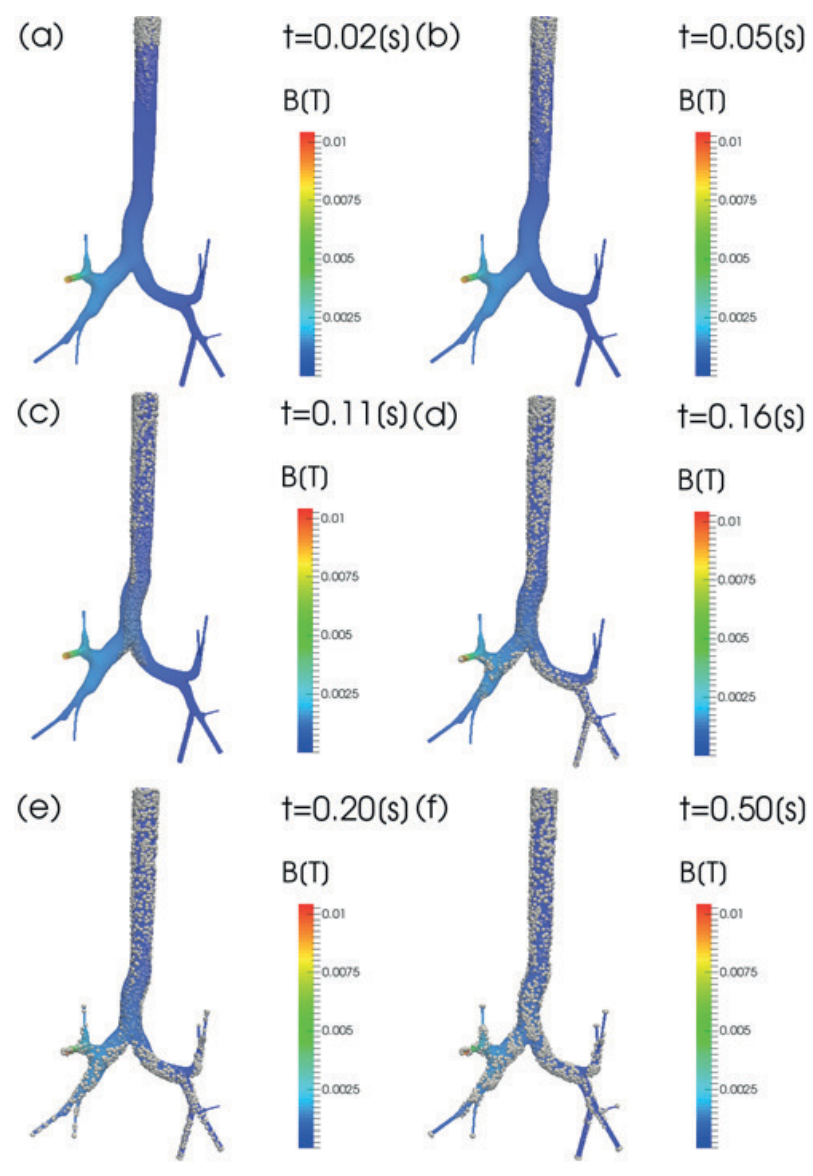

Figure 6: Magnetic Induction Field and particle positions at different time steps, with external magnetic field. 


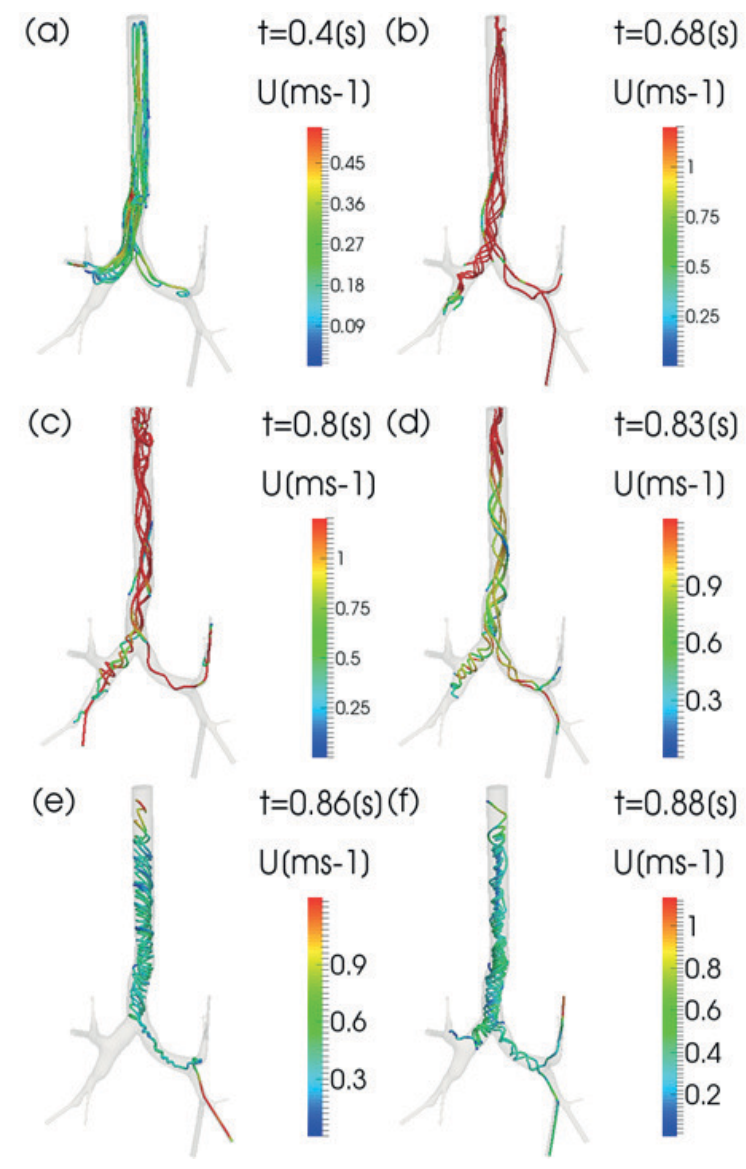

Figure 7: Velocity Field and velocity streamlines at different time steps with external magnetic field. 

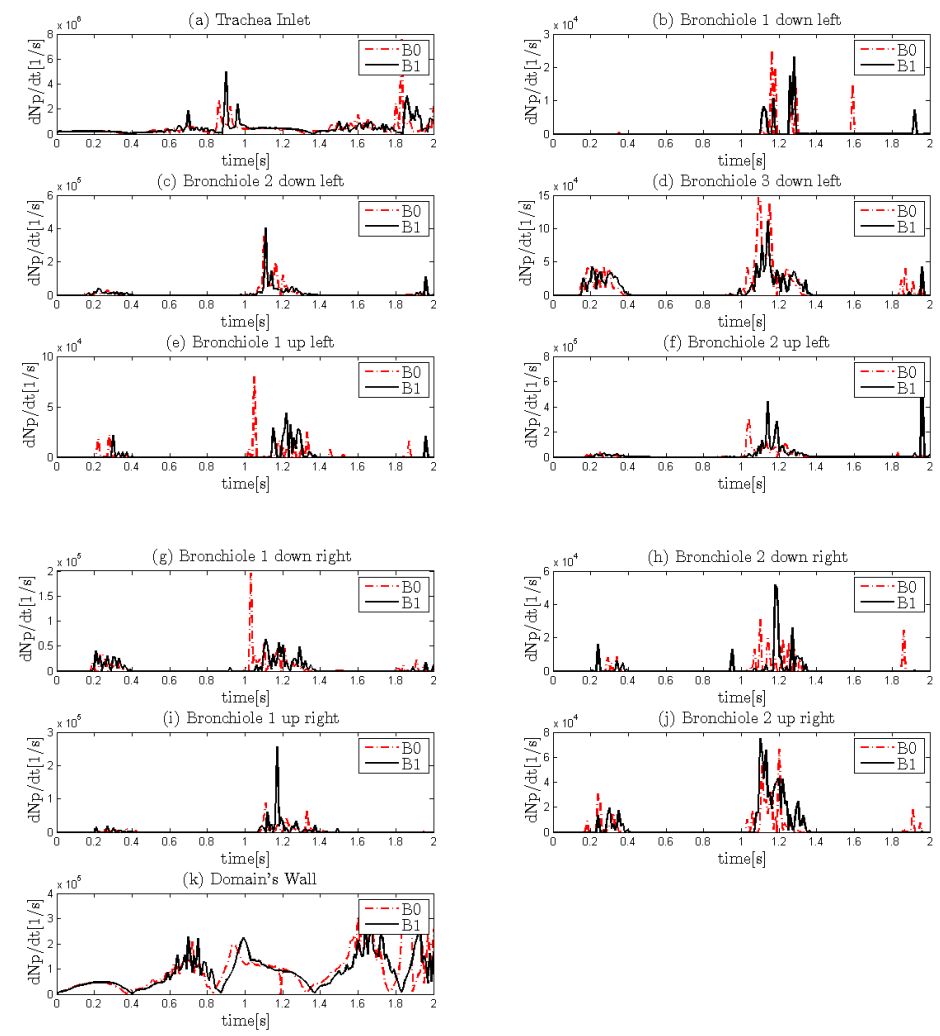

Figure 8: Particle volumetric flow rate per unit volume vs time for the two different conditions. $\mathrm{B} 0=$ magnetic field off; $\mathrm{B} 1$ = magnetic field on . 

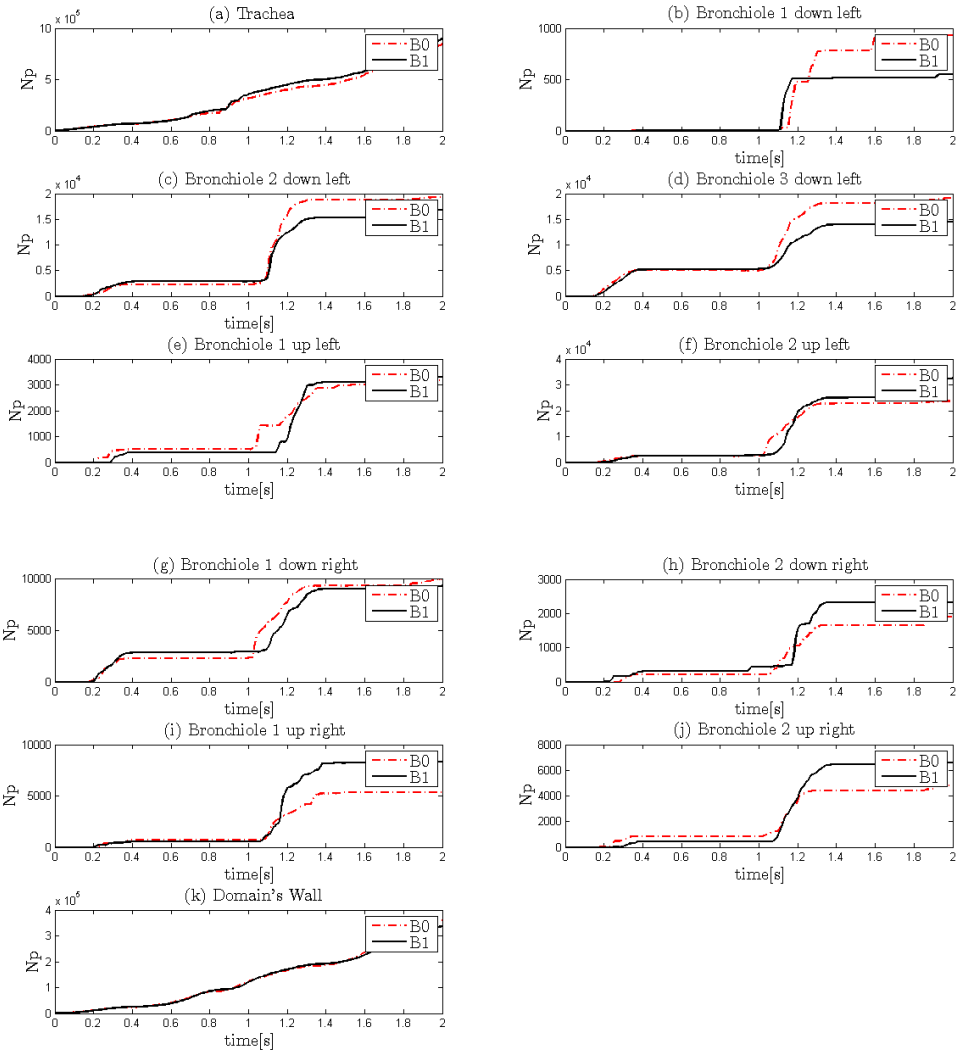

Figure 9: Particle number vs time for the two different conditions. $\mathrm{B} 0=$ magnetic field off; $\mathrm{B} 1$ = magnetic field on. 